Ann. Sci. forest., 1967, 24 (2), 107-119.

\title{
ÉTUDE DE LA LONGUEUR DES FIBRES SUR ÉCHANTILLONS PRÉLEVÉS A LA TARIÈRE DE PRESSLER DE 5 MM DE DIAMÈTRE
}

\author{
H. POLGE, C. MILLIER \\ Station de Recherches sur la Qualité des Bois, \\ Station de Biométrie, \\ Centre national de Recherches forestières, 54 - Nancy \\ Institut national de la Recherche agronomique
}

SOMMAIRE

Les études de longueur de fibres sur des arbres vivants étaient jusqu'ici réalisées sur échantillons prélevés avec des tarières de fort diamètre qui ne pouvaient être enfoncées qu'à l'aide d'un moteur ou d'un étrier auxiliaire.

Il est proposé ici d'utiliser des prélèvements obliques par rapport au fil du bois, mais obtenus avec des tarières standards de $5 \mathrm{~mm}$ et d'enlever sur ces échantillons les deux dosses latérales à l'aide d'une scie circulaire spécialement conçue à cet effet.

De la sorte, le pourcentage de fibres intactes peut être deux fois plus élevé qu'avec un diamètre de $10 \mathrm{~mm}$ utilisé perpendiculairement au fil du bois.

\section{1. - GÉNÉRALITÉS}

L'étude de la valeur papetière comparée des diverses essences ou provenances, de son héritabilité ou de l'influence qu'exercent sur elle les différents facteurs de l'environnement prend à l'heure actuelle de plus en plus d'importance dans le monde en raison de l'augmentation continue des besoins en pâtes de bonne qualité. 
Or, l'aptitude papetière est régie directement par les caractéristiques biométriques des fibres utilisées, et tout particulièrement par leur longueur, qui ne peut être déterminée aisément qu'en procédant au défibrage d'un échantillon de bois de dimensions appropriées.

Malheureusement, ces caractéristiques sont sujettes à une telle variabilité interne et entre individus qu'il faut, pour en obtenir des valeurs moyennes statistiquement valables, analyser un nombre élevé d'échantillons, ce qui n'est possible que si l'on peut se les procurer par des procédés non destructifs, c'est-à-dire sans qu'il soit nécessaire d'abattre les arbres en cause.

L'utilisation de (( carottes de sondage ) prélevées à la tarière sur des arbres vivants paraîtrait donc, à première vue, toute indiquée si, par malchance, l'ordre de grandeur de la longueur des fibres n'était, chez les résineux tout au moins, voisin du diamètre des tarières les plus couramment utilisées en matière de recherches forestières, celles de $5 \mathrm{~mm}$.

Quand, par suite, on cherche à étudier la longueur des fibres en employant de telles tarières suivant les techniques habituelles, on se heurte à de sérieuses difficultés dues à une proportion très élevée (pouvant atteindre $100 \%$ ) de fibres coupées ; les rares fibres intactes à mesurer doivent être recherchées au prix de pertes de temps considérables, au milieu d'une accumulation d'éléments plus ou moins fractionnés ; de plus, la probabilité de sectionner une fibre étant d'autant plus grande que sa longueur est plus élevée, les diagrammes de fréquence sont faussés, les effectifs des classes de longueurs supérieures à la moyenne étant sous-évalués par rapport aux autres.

C'est en s'appuyant sans doute sur de telles raisons que la très officielle FAO (1963) a pu écrire, dans une de ses notes sur l'Equipement forestier, que: ( les tarières standards... ne permettent pas des mesures suffisamment précises de la longueur des fibres... ).

\section{2. - MATÉRIEL ET MÉTHODES DE PRÉLÈVEMENT POSSIBLES}

\section{1. - Utilisation de tarières de fort diamètre}

Pour pallier le double inconvénient mentionné ci-dessus, l'usage de tarières de fort diamètre (10 $\mathrm{mm}$ en général) se répand de plus en plus, notamment dans les pays anglo-saxons. Malheureusement, la mise en œuvre de telles tarières, outre les traumatismes plus importants qu'elle occasionne aux arbres en cause, ne peut pratiquement pas être assurée par la seule force musculaire des opérateurs.

Pour être à même de les utiliser, il faut, soit recourir à une source motrice extérieure, comme c'est le cas pour la tarière mécanique australienne faisant l'objet de la figure 1, soit fixer au préalable sur l'arbre à sonder un étrier muni d'un filetage intérieur sur lequel on visse la tarière, assurant du même coup son enfoncement à l'intérieur du bois (voir sur la figure 2 un tel dispositif s'inspirant d'un prototype mis au point par la Station de Recherches de la Forestry Commission britannique).

Il faut bien dire que ni l'un ni l'autre de ces procédés n'est pleinement satisfaisant car tous deux nécessitent l'emploi d'un matériel lourd et encombrant, dont la mise en place exige des délais importants et ralentit considérablement les opérations de prélèvement. 


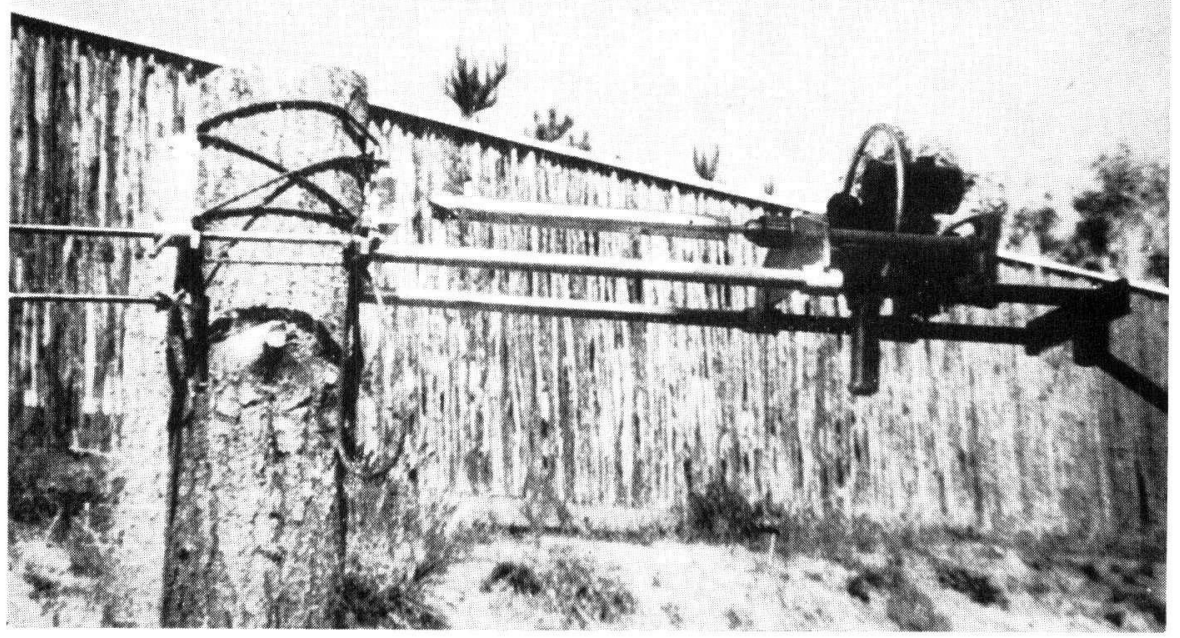

FIg. 1. - Tarière mécanique australienne

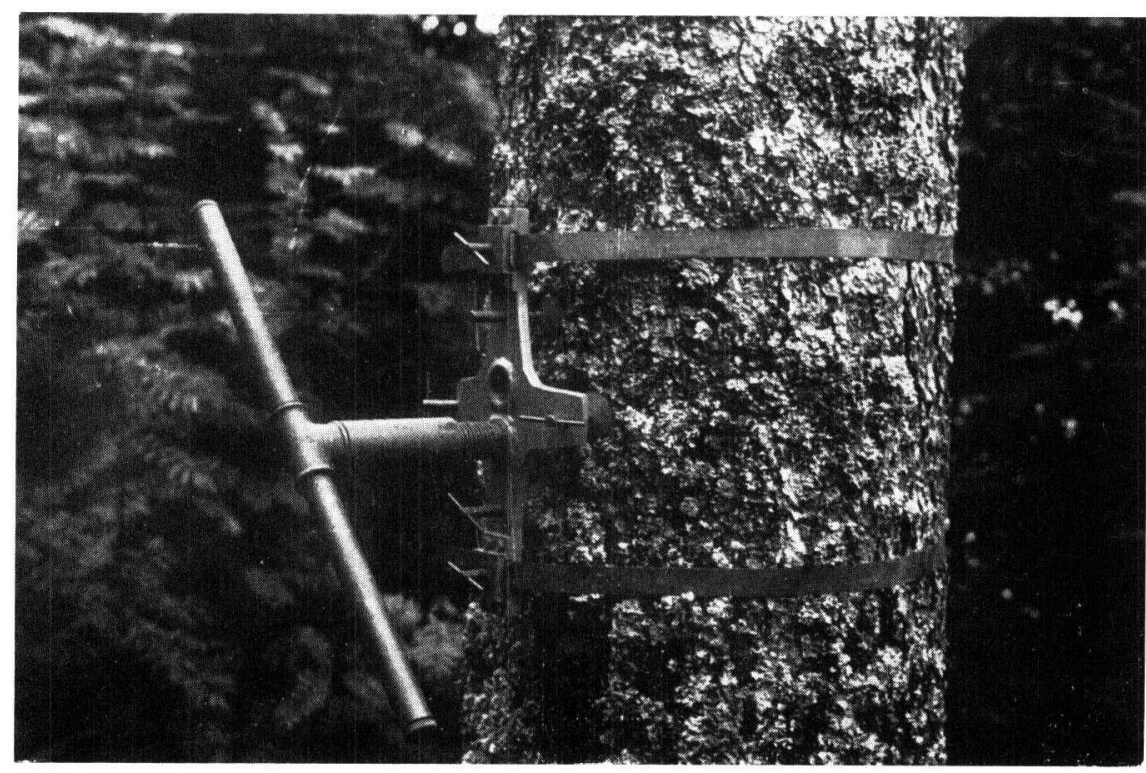

FIG. 2. - Etriers pour la mise en place des tarières de $10 \mathrm{~mm}$ de diamètre

\section{2. - Utilisation de tarières normales de $5 \mathrm{~mm}$}

\subsection{1. - Réalisation de prélèvements obliques}

La Station de Recherches sur la Qualité des Bois du C.N.R.F. a pour sa part cherché à obtenir, à l'aide de tarières traditionnelles de $5 \mathrm{~mm}$ de diamètre, les mêmes facilités au point de vue étude 
des longueurs de fibres qu'avec des échantillons de $10 \mathrm{~mm}$ de diamètre, sans avoir à subir les inconvénients inhérents à l'utilisation d'un moteur ou d'un étrier accessoire.

Le moyen retenu pour y parvenir est extrêmement simple, puisqu'il consiste à procéder, avec une tarière ordinaire actionnée manuellement, à un prélèvement oblique par rapport au fil du bois (fig. 3).

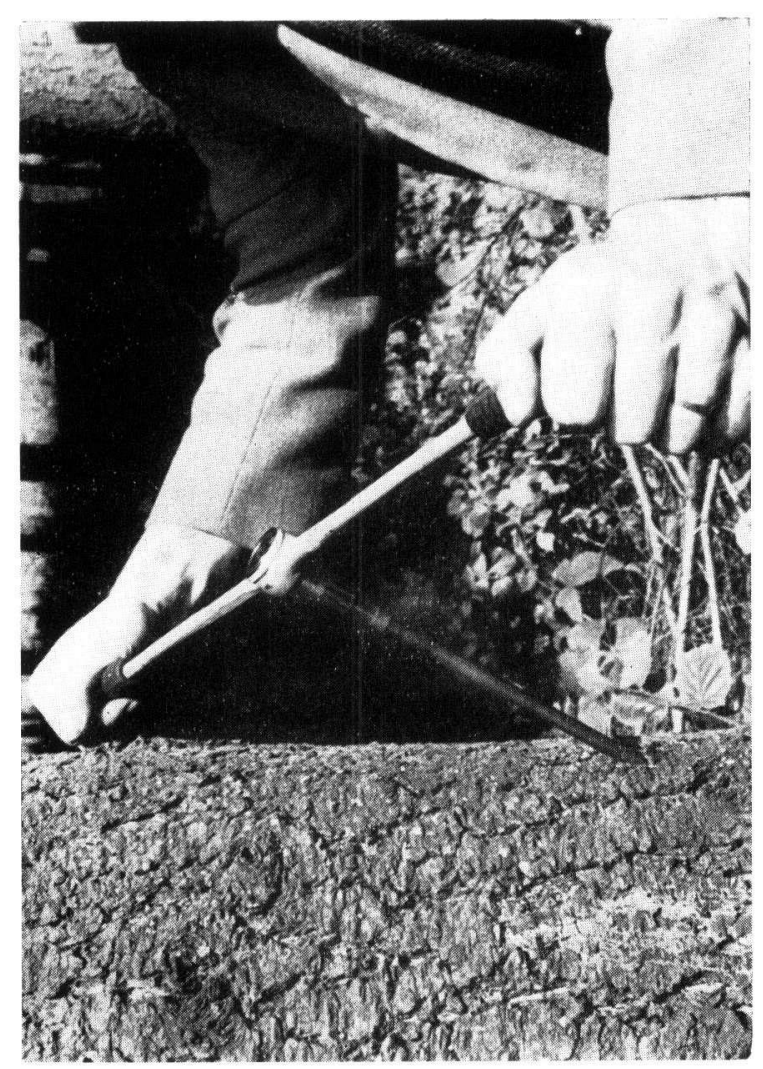

F:G. 3. - Prélèvement oblique à l'aide d'un manche à cliquets

La seule difficulté tient au fait que, dans de telles conditions, il n'est pas possible de faire faire au manche un tour complet, dès lors que sa demi-longueur excède la distance qui sépare la tête de la tarière du tronc de l'arbre suivant une perpendiculaire à l'axe; mais cette difficulté peut être facilement tournée en utilisant un dispositif à cliquets qui permet de faire pénétrer la totalité de la tarière dans l'arbre, facilement et sans fatigue, par un simple mouvement de va-et-vient.

On peut extraire de la sorte des carottes de sondage aussi peu inclinées que l'on veut par rapport à l'axe des fibres, et dans lesquelles chaque limite d'accroissement annuel est représentée par une ellipse dont le grand axe (qui correspond à la direction longitudinale de l'arbre) est égal au diamètre de la tarière divisé par le sinus de l'angle d'inclinaison ; ainsi obtient-on, avec une tarière de $5,4 \mathrm{~mm}$ de diamètre et un angle de $30^{\circ}$, une dimension maximale parallèle au fil du bois de l'ordre de $10,8 \mathrm{~mm}$ (voir sur la figure 4 la radiographie d'une telle carotte prise dans un Pin sylvestre).

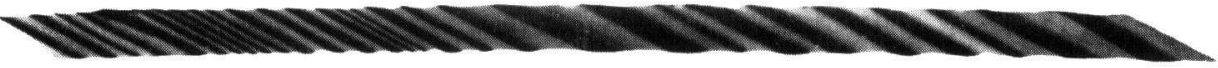

Fig. 4. - Radiographie de carotte de Pin sylvestre prélevée en oblique par rapport au fil du bois (grandeur nature) 
Quelques précautions simples doivent cependant être respectées pour mener à bien ce genre de prélèvement.

Il faut tout d'abord utiliser des tarières parfaitement affûtées car le bois est plus facilement sectionné perpendiculairement à la direction des fibres qu'en oblique par rapport à elle.

Il est également nécessaire, surtout dans les bois très résineux, de bien nettoyer l'intérieur des tarières ; à défaut, la carotte de sondage, au lieu de rester libre dans l'axe de l'évidement tronconique interne, a tendance à coller sur une des génératrices et à être plus ou moins vissée sur elle-même, ce qui a pour effet d'en augmenter localement le diamètre, d'entraîner un engorgement de l'extrémité et de rendre, par suite, très difficile la récupération de l'échantillon.

Enfin, plus encore peut-être que pour des prélèvements normaux, il convient de reboucher le trou de tarière avec une cheville de bois antiseptisée afin d'éviter une dépréciation ultérieure trop importante des produits.

\subsection{2. - Débit des carottes de sondage}

Les carottes obtenues de cette façon présentent encore une proportion élevée de fibres coupées dès lors que l'on s'écarte trop du plan de symétrie défini par l'axe du prélèvement et l'axe de fibre.

Pour remédier à cette situation, la Station a mis au point un système de scie circulaire miniature utilisant des lames de faible épaisseur $(0,5 \mathrm{~mm})$ et à voie nulle, tournant à $2100 \mathrm{tr} / \mathrm{mn}$. L'échantillon cylindrique est serré à l'aide de mâchoires spécialement conçues à cet effet sur un chariot qui coulisse lui-même sur une platine de microscope, ce qui permet d'en régler la position au $1 / 10$ de mm près (fig. 5). On peut ainsi enlever à la carotte de sondage les deux dosses latérales qui comportent beaucoup de fibres coupées pour n'en conserver que la partie centrale sur une épaisseur de 1,5 mm environ.

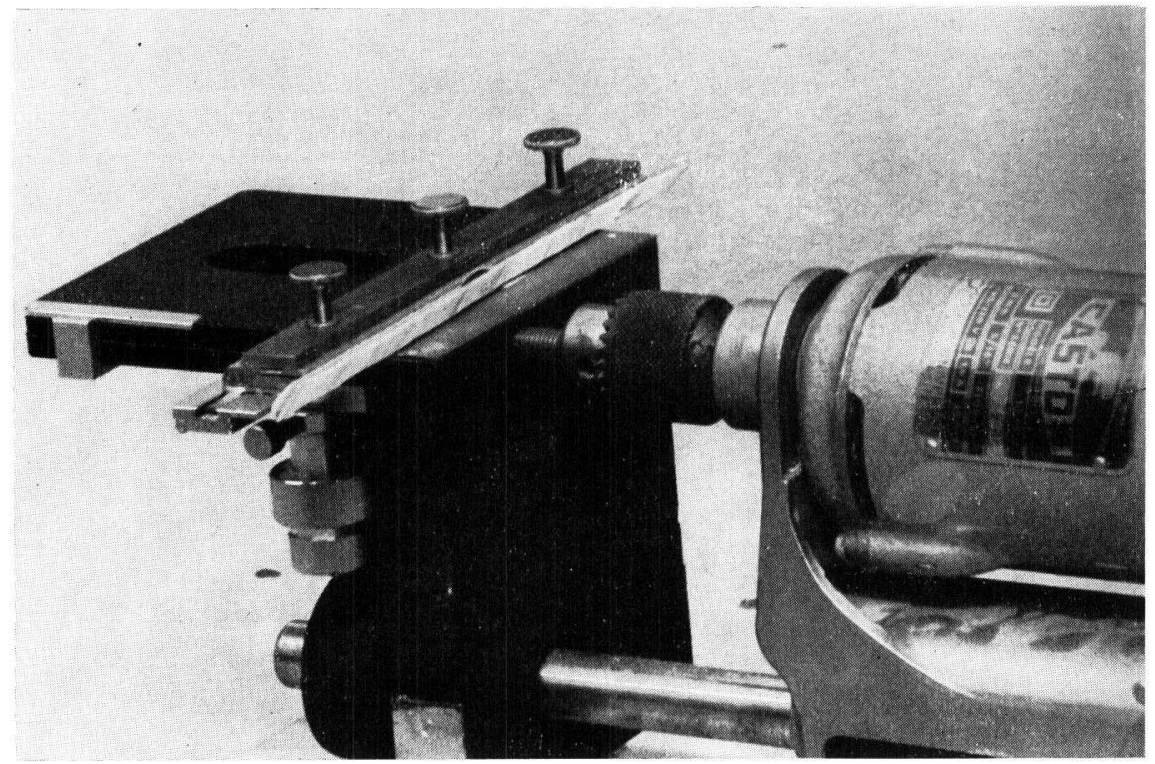

FIG. 5. - Scie circulaire pour débitage longitudinal des carottes de sondage de $5 \mathrm{~mm}$

Le calcul montre que, si la dimension maximale parallèlement à l'axe de fibre est, dans le plan de symétrie, de 10,8 par exemple, elle ne tombe sur les bords de l'échantillon ainsi débité qu'à $10,4 \mathrm{~mm}$, ce qui est encore très supérieur à la longueur des fibres de n'importe quelle essence forestière.

Sans doute les dimensions tangentielles d'un tel échantillon peuvent-elles paraître très faibles, mais cela importe peu puisqu'il n'existe pas de variations notables de la longueur des fibres dans cette direction. 
Cette faible épaisseur devient au contraire un avantage lorsqu'on souhaite se livrer à des études biométriques fines, cerne par cerne, ou même fraction de cerne par fraction de cerne ; il est notamment très facile de procéder ainsi à des déterminations de longueur de fibre pour le bois de printemps ou le bois d'été d'une année donnée; la séparation des diverses zones d'accroissements peut en effet se faire sans précaution spéciale avec une simple lame de canif sur des échantillons d'épaisseur aussi réduite, alors qu'il conviendrait, au contraire, en toute rigueur, avec des échantillons plus épais, de procéder à un découpage suivant des trajets concaves pour tenir compte de la courbure des cernes qui ne peut être négligée, surtout à proximité de la moelle.

Au reste, un fragment de carotte de $1,5 \mathrm{~mm}$ d'épaisseur dans le sens tangentiel et de $0,5 \mathrm{~mm}$ seulement dans le sens radial, ce qui représente vraiment un cas extrême, renferme encore un minimum de l'ordre de 300 alignements de fibres dans un échantillon de bois de printemps de résineux, et de plus de 600 dans le bois d'été ; on peut donc largement y procéder aux cinquante mesures individuelles généralement requises pour obtenir une longueur moyenne statistiquement valable.

\subsection{3. - Défibrage}

Enfin, lorsqu'on désire établir un diagramme de fréquence des longueurs de fibre pour l'ensemble des accroissements annuels d'un arbre à un niveau donné, la réalisation de tels échantillons permet d'obtenir un défibrage facile sans autre manipulation supplémentaire, puisque les lessives utilisées pénètrent facilement à l'intérieur d'éprouvettes d'aussi faible épaisseur.

On peut, en particulier, suivant une méthode déjà décrite (PoLGE, 1963), suggérée à l'origine par J. Chiaverina de l'Ecole $f$ ançaise de Papeterie et perfectionnée récemment à la Station, placer directement les échantillons dans toute une série de tubes d'acier munis d'un joint en plomb (comme le modèle représenté sur la fizure 6) et remplis d'un mélange de soude et̂ de monosulfure de sodium ; on range ensuite tous ces tubes dans un panier, qui est animé d'un mouvement de rotation à la vitesse de $1 \mathrm{tr} / \mathrm{mn}$, à l'intérieur d'une étuve portée à $160^{\circ}$ (fig. 7) ; on réalise ainsi à très petite échelle une cuisson très voisine de celle du procédé Kraft, et on obtient en une seule opération, et dans des conditions rigoureusement comparables, le défibrage d'un grand nombre d'échantillons sur lesquels les caractéristiques biométriques peuvent, par la suite, être étudiées sans difficulté (*).

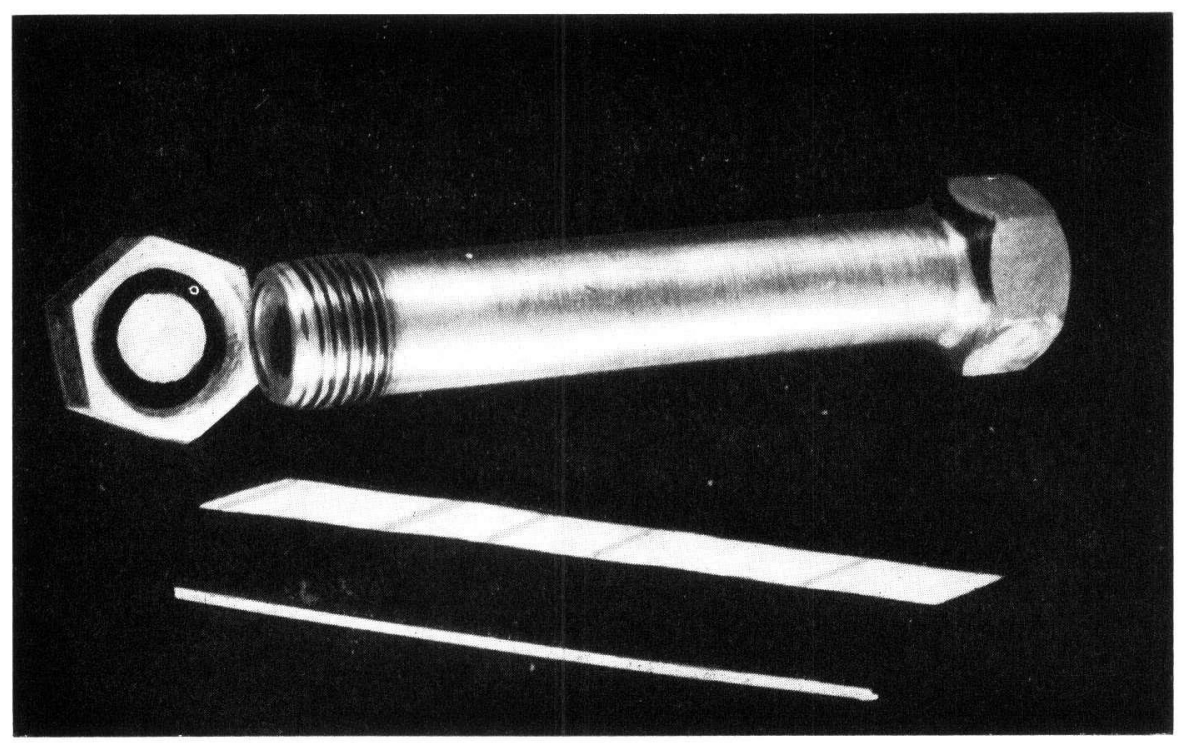

FIG. 6. - Lessiveur de laboratoire pour défibrage d'échantillons minces

* La commande à cliquets des tarières, la scie miniature montée sur platine de microscope et le panier de défibrage rotatif ont été réalisés dans la pratique par M. COCHARD, Technicien à la Station de Recherches sur la Qualité des Bois, que nous tenons à remercier bien vivement ici. 


\section{3. - ÉTUde MATHÉMATIQUE COMPARÉE DES DEUX PROCÉdÉS}

Il est possible, moyennant un certain nombre d'hypothèses tout à fait justifiables, de calculer mathématiquement le pourcentage de fibres intactes contenu, d'une part dans un prélèvement perpendiculaire au fil du bois réalisé avec une tarière de fort diamètre, d'autre part dans un prélèvement oblique effectué avec une tarière ordinaire, mais débité comme il est indiqué ci-dessus.

Ce calcul peut être fait aussi bien dans le cas de fibres de longueur uniforme que dans celui de fibres dont la dimension longitudinale suit une loi de répartition normale et nécessite la détermination préalable du pourcentage de fibres intactes contenu dans un échantillon parallélépipédique de dimensions données.
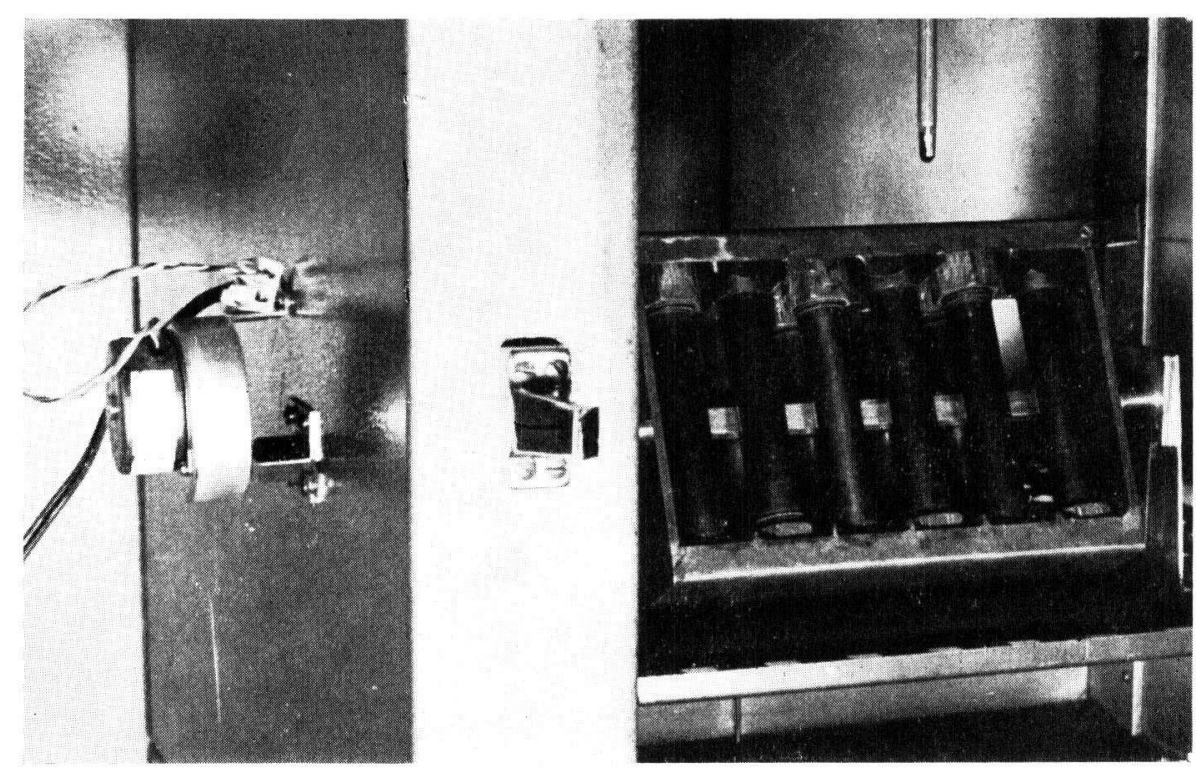

FIG. 7. - Panier de défibrage rotatif

\section{1. - Les hypothèses préliminaires}

La distribution des fibres dans le bois se fait suivant les modalités ci-après :

- la direction des fibres est fixe,

- les fibres sont réparties suivant une loi de probabilité uniforme,

- leur longueur obéit à des lois déterminées (on considère d'abord la loi uniforme puis la loi normale),

- la distribution des fibres et celle de leur longueur sont indépendantes. 
Par suite de la forme fusiforme des fibres, une fibre de longueur $l$ dont une partie de longueur au moins égale à $l-e$ est comprise dans l'échantillon, est considérée comme intacte, $e$ étant une valeur donnée a priori par l'expérimentateur.

Donc, si on tient compte de cette modification, la proportion de fibres intactes de longueur $l$ sera égale à la valeur que prendra la formule donnant la proportion des fibres intactes quand on ne tient pas compte de la modification pour $l-e$.

\section{2. - Définition de la loi normale des longueurs}

Les longueurs obéissent à une loi normale tronquée en $l=0$ étant donné que les longueurs ne peuvent être que positives. La moyenne est $L$, l'écart type $s$.

La fonction de répartition est :

$$
F_{1}(l)=\int_{0}^{l} f_{1}(t) \mathrm{d} t \text { avec } f_{1}(t)=\frac{1}{\sqrt{2 \pi s}} \frac{1}{1-F_{1}(0)} e^{-\frac{1}{2}\left(\frac{t-L}{s}\right)^{2}}
$$

en se ramenant à la variable normale réduite de fonction de répartition $F(x)$ et de densité $f(t)$, on a :

$$
F_{1}(l)=\frac{F\left(\frac{l-L}{s}\right)-F\left(-\frac{L}{s}\right)}{1-F\left(-\frac{L}{s}\right)} \text { avec } f(t)=\frac{1}{\sqrt{2 \pi}} \frac{1}{1-F\left(-\frac{L}{s}\right)} e^{-t^{2 / 2}} .
$$

En réalité, comme on le verra dans les applications numériques,

$$
-\frac{L}{s}<-5, \frac{l-L}{s}>5, \text { alors } F_{1}(l)=1
$$

par suite des faibles probabilités attachées à ces queues de distribution.

De même :

$$
J_{1}\left(\frac{l-L}{s}\right)=\int_{-\frac{L}{s}}^{\frac{l-L}{s}} t f(t) \mathrm{d} t=0,=J_{2}\left(\frac{l-L}{s}\right)=\int_{-\frac{L}{s}}^{\frac{l-L}{s}} t^{2} f(t) \mathrm{d} t=1 .
$$

3.3. - Nombre de fibres dans un parallélépipède de base rectangulaire où un côté est parallèle aux fibres

Pour résoudre le problème, on a besoin d'exprimer la proportion de fibres intactes dans un tel solide.

Le nombre de fibres présentes en tout ou en partie dans le rectangle de côtés $H$ 
et dy est le nombre de fibres dont l'extrémité inférieure est contenue dans le rectangle de côtés $H+l$ et dy (fig. 8).

FIG. 8

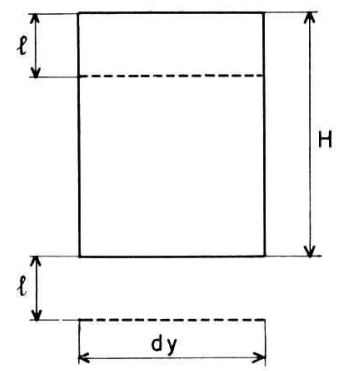

Alors de façon évidente, on a :

$$
\begin{gathered}
\operatorname{Pr}\{\text { fibres intactes }\}=P_{00}=\frac{H-l}{H+l} \text { si } l<H \\
P_{00}=0 \quad \text { si } l \geqslant H
\end{gathered}
$$

3.4. - Nombre de fibres intactes dans un cylindre à section parallèle aux fibres de forme ellipsoïde tronquée

Soit $r$ la densité des fibres, $a$ la longueur du petit axe, $b$ celle du grand axe, $\delta$ la demi-épaisseur (suivant le petit axe) de la partie conservée, $l$ la longueur des îbres, $L$ la longueur moyenne, $s$ l'écart type..., $\psi$ l'angle d'inclinaison de la tarière.

$x$ et $y$ sont des coordonnées cartésiennes, $\phi$ un paramètre permettant de définir le point courant de l'ellipse.

En appliquant les résultats de 3.3, on décompose la surface en petits rectangles élémentaires et on intègre (fig. 9).

FIG. 9

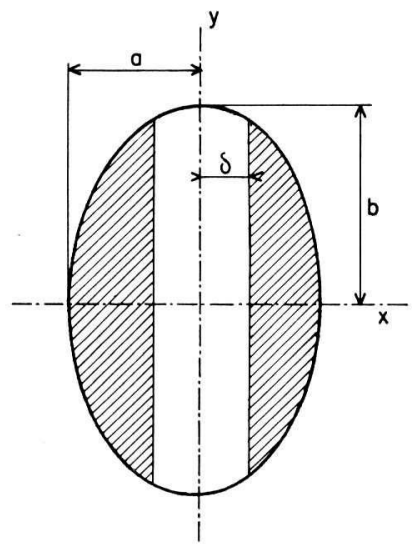




\subsection{1. - Fibres de longueur constante}

L'équation paramétrique de la demi-ellipse est :

$$
\text { (E) }\left\{\begin{array}{l}
x=a \cos \phi \\
y=b \sin \phi
\end{array} 0<\phi<\pi\right.
$$

Toutes les fibres de l'échantillon ont leur extrémité inférieure dans une surface délimitée par :

(E) au-dessus de l'axe des $x$

(L) au-dessous avec

$$
\text { (L) }\left\{\begin{array}{l}
x=a \cos \phi \\
y=-b \sin \phi-l
\end{array} \quad 0<\phi<\pi\right.
$$

Leur nombre sera égal (au signe près) à :

$$
I_{1}=r \int_{\alpha}^{\beta}-a \sin \phi(2 b \sin \phi+l) \mathrm{d} \phi
$$

et le nombre des fibres intactes à :

$$
I_{2}=r \int_{\alpha}^{\beta}-a \sin \phi\left(\frac{2 b \sin \phi-l}{2 b \sin \phi+l}\right)(2 b \sin \phi+l) \mathrm{d} \phi
$$

avec

$$
\alpha=\frac{\pi}{2}-\operatorname{Arcsin} \frac{\delta}{a}, \quad \beta=\frac{\pi}{2}+\operatorname{Arcsin} \frac{\delta}{a}
$$

d'où la proportion de fibres intactes

$$
p_{10}(l)=\frac{b\left(\operatorname{Arcsin} \frac{\delta}{a}+\frac{\delta}{a} \sqrt{\left.1-\frac{\delta^{2}}{a^{2}}\right)}-\frac{l-}{a}\right.}{b\left(\operatorname{Arcsin} \frac{\delta}{a}+\frac{\delta}{a} \sqrt{\left.1-\frac{\delta^{2}}{a^{2}}\right)+l-\frac{\delta}{a}}\right.} .
$$

\subsection{2. - Fibres de longueur normale}

D'après les théorèmes fondamentaux des probabilités, $P_{11}=\operatorname{Pr}\{$ fibre intacte $\}=\Sigma\left[P_{r}\right.$ ffibre intacte si longueur $\left.=l\right\} \cdot \operatorname{Pr}\{$ longueur $\left.=l\}\right]$

$$
P_{11}=\int_{0}^{2 b} p_{10}(l) f_{1}(l) \mathrm{d} l
$$

on développe $p_{10}(l)$ au voisinage de $l=L$ et on intègre ensuite. Cela est possible d'après les régularités des fonctions. 
FIG. 10

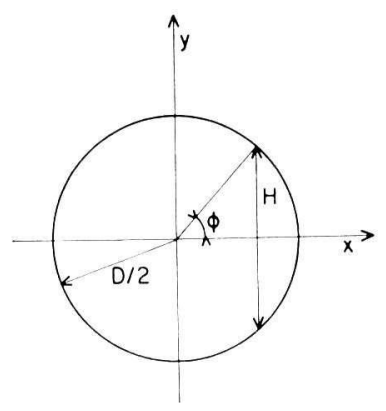

On se limite au deuxième ordre et on intègre en tenant compte du fait que :

$$
J_{1} \frac{2 b-L}{s}=0, J_{2} \frac{2 b-L}{s}=1
$$

et

$$
F_{1}(2 b)=1
$$

On trouve en posant :

$$
\begin{gathered}
t=\operatorname{Arcsin} \frac{\delta}{a}+\frac{\delta}{a} \sqrt{1-\frac{\delta^{2}}{a^{2}}} \\
p_{11}=p_{10}(L)+\frac{2 t\left(\frac{\delta}{a}\right)^{2} b s^{2}}{\left(t b+\frac{\delta}{a} L\right)^{3}} .
\end{gathered}
$$

3.5. - Nombre de fibres intactes dans un cylindre à section circulaire parallèle aux fibres

Soit $r$ la densité des fibres, $D$ le diamètre du cercle $(c), \phi$ l'angle de paramétrage, $l$ la longueur des fibres, $L$ la longueur moyenne, $s$ l'écart type, $k=l / D, K=L / D$, $H$ la longueur d'une corde, $x$ et $y$ sont les coordonnées cartésiennes (fig. 10).

On effectue les mêmes raisonnements de départ qu'en 3.4.

\subsection{1. - Longueur des fibres constantes}

Le nombre de fibres présentes est

$$
I_{1}=-r \frac{\pi D^{2}}{4}\left(1+\frac{4 k}{\pi}\right) .
$$

Il ne peut y avoir de fibres de longueur $l$ intactes que si $l<H$, or ici, $H=D \sin \phi$ donc :

$$
\operatorname{Arcsin} k<\phi<\pi-\operatorname{Arcsin} k
$$


Le nombre de fibres intactes est donc :

$$
I_{2}=r \int_{\operatorname{Arcsin} K}^{\pi-\operatorname{Arcsin} k} \frac{D \sin \phi-l}{D \sin \phi+l} \frac{D}{2} \sin \phi(D \sin \phi+l) \mathrm{d} \phi .
$$

On trouve finalement comme proportion de fibres intactes :

$$
p_{20}=\frac{\pi-2 \operatorname{Arcsin} k-2 k \sqrt{1-k^{2}}}{\pi+4 k} .
$$

\subsection{2. - Fibres de longueur normale}

La proportion de fibres intactes est :

$$
p_{21}=\int_{-\frac{L}{s}}^{\frac{D-L}{s}} f(y) g(y) \mathrm{d} y
$$

avec

$$
g(y)=\frac{\pi-2 \operatorname{Arcsin}\left(K+\frac{s}{D} y\right)-2\left(K+\frac{s}{D} y\right) \sqrt{1-\left(K+\frac{s}{D} y\right)^{2}}}{\pi+4 K+4 \frac{s}{D} y}
$$

et le $f(y)$ du paragraphe 3.2. On développe $g(y)$ jusqu'au deuxième ordre au voisinage de 0 , on obtient en intégrant (ceci étant possible grâce aux régularités des fonctions),

$$
p_{21}=p_{20}+\frac{s^{2}}{D^{2}} \times \frac{2}{(\pi+4 K)^{2}}\left(\frac{K(\pi+4 K)}{\sqrt{1-K^{2}}}+8\left(p_{20}+\sqrt{\left.1-K^{2}\right)}\right) .\right.
$$

\section{6. - Application numérique}

On prend les données réelles suivantes :

$D=10 \mathrm{~mm}, a=2,7 \mathrm{~mm} ; b=\frac{a}{\sin \psi}, \psi=\frac{\pi}{6}, \quad b=5,4 \mathrm{~mm}, \quad l=3,5 \mathrm{~mm}$; $s=0,3 \mathrm{~mm} ; \delta=0,75 \mathrm{~mm}$.

On trouve :

$$
\begin{array}{ll}
p_{20}=0,245 \quad \begin{array}{l}
\text { (proportion de fibres non coupées (longueur constante) dans un } \\
\text { cylindre à section circulaire). }
\end{array} \\
p_{21}=0,245+\begin{array}{l}
0,0007=0,246 \\
\text { (proportion de fibres non coupées (longueur normale) dans un } \\
\text { cylindre à section circulaire). }
\end{array} \\
\begin{array}{l}
\text { (proportion de fibre non coupées (longueur constante) dans } \\
p_{10}=0,505 \text { cylindre à section ellipsoïde). }
\end{array} \\
p_{11}=0,505+\begin{array}{l}
0,001 \\
\text { (proportion de fibres non coupées (longueur normale)). }
\end{array}
\end{array}
$$


L'influence de la normalité est donc pratiquement négligeable à condition que le coefficient de variation $s / L$ soit petit (il est ici de $8,6 \%$ ).

On voit que la proportion de fibres intactes est plus de deux fois plus élevée dans un échantillon prélevé en oblique avec une tarière de $5 \mathrm{~mm}$ et débité suivant la procédure indiquée plus haut que dans une carotte de $10 \mathrm{~mm}$ de diamètre prise perpendiculairement au fil du bois.

Reçu pour publication en mai 1967

\author{
SUMMARY \\ STUDIES OF FIBRELENGTH WITH INCREMENT CORES OF 5 MM
}

Instead of using large diameter increment cores to study fibrelength from living tree (which often is only feasible with mechanized equipment or by placing a clamp on the tree), it is suggested to use $5 \mathrm{~mm}$ increment cores slanting to the grain direction and to cut off two lateral slabs with an appropriate circular saw. Thus the percentage of uncut fibres can be two times higher than within a $10 \mathrm{~mm}$-core normally taken.

\title{
ZUSAMMENFASSUNG
}

UNTERSUCHUNG DER FASERLÄNGE VON HOLZPROBEN DIE MIT DEM PRESSLER'SCHEN ZUWACHSBOHRER (BOHRKERNDURCHMESSER 5 MM) ENTNOMMEN WURDEN

Bei Untersuchungen der Faserlänge an lebenden Bäumen wurden bisher meist Zuwachsbohrer mit einem grossen Bohrkerndurchmesser verwendet, welche entweder einen Motorantrieb besitzen oder durch eine besondere Hilfsvorrichtung am Baum befestigt werden müssen.

In der vorliegenden Arbeit wird vorgeschlagen Bohrkerne von nur $5 \mathrm{~mm}$ Durchmesser mit einem Pressler'schen Zuwachsbohrer schräg zur Holzfaserrichtung zu entnehmen und anschiessend die beiden parallel zur Faserrichtung liegenden Segmente mit einer eigens zu diesem Zwecke entwickelten Kreissäge abzuschneiden.

Der mit dieser Methode erzielte Anteil an unverletzten Fasern ist doppelt so gross als jener, der bei einer Probenentnahme von $10 \mathrm{~mm}$ Durchmesser, senkrecht zur Faserrichtung, erreicht werden kann.

\section{RÉFÉRENCES BIBLIOGRAPHIQUES}

FAO, 1963. Une tarière de Pressler à moteur. Note sur l'Equipement forestier, n A 3063.

Polge H., 1963. Contribution à l'étude de la qualité du bois des principales essences résineuses exotiques utilisées dans les reboisements français. Ann. Ec. nation. Eaux et Forêts et Stn Rech. Exper., 20, 3, pp. 403-469. 